\title{
Análisis de esfuerzo máximo de compresión y flexión de tres tipos de cementos refractarios utilizados en calderas
}

\author{
Karla María Romero Reyes ${ }^{1}$, Carlos Francisco Irías Caballero ${ }^{2}$, Carlos José \\ SÁNChez CÁrCAmo ${ }^{3}$, ANd Gerhard Rummel ${ }^{4}$ \\ ${ }^{1}$ Escuela de Física - UNAH, mail:karlamromero10803@gmail.com \\ ${ }^{2}$ Escuela de Física - UNAH, mail:carlosirias18@hotmail.com \\ ${ }^{3}$ Escuela de Física - UNAH, mail:carlos2014sanchez25@gmail.com \\ ${ }^{4}$ Escuela de Física - UNAH, mail:gerharunah@gmail.com
}

Recibido: 08 de Febrero de 2016 / Aceptado: 01 de Mayo de 2016

\begin{abstract}
Resumen
The proper selection of refractory cements used in industrial boilers and furnaces, depends on the criteria of quality, improvement and verification of mechanical, thermal and chemical properties. Our work is based on obtaining bending mechanical properties (MOR, Modulus Of Rupture) and compression (CCS, Cold Crushing Strength) conducted at room temperature for three types of refractory concrete.
\end{abstract}

Keywords: Refractory, Strength and Quality.

La selección adecuada de cementos refractarios utilizados en calderas y hornos industriales, depende de los criterios de calidad, mejoramiento y verificación de las propiedades mecánicas, térmicas y químicas. Nuestro trabajo se basa en la obtención de las propiedades mecánicas de flexión (MOR, Modulus Of Rupture) y de compresión (CCS, Cold Crushing Strength) realizadas a temperatura ambiente para tres tipos de cementos refractarios.

Palabras clave: Refractario, Resistencia y Calidad.

\section{INTRODUCCIÓN}

$\mathrm{P}$ RESEnTAmos el estudio experimental del análisis del esfuerzo máximo de compresión, y de flexión de tres tipos de cementos refractarios utilizados en calderas de la industria azucarera de Honduras, el cual posee un impacto económico y social en el país.

Es de suma importancia para un desarrollo económico eficiente, el conocer y estudiar materiales como los cementos refractarios. Midiendo su resistencia a compresión y tracción se obtienen criterios que permiten un mejor aprovechamiento en la aplicación industrial.

Un buen cemento refractario debe proporcionar buena resistencia a los embates del medio ambiente al que es expuesto, debe tener baja conductividad térmica para hacer más eficiente el uso del calor dentro de la caldera, aminorando las pérdidas de calor hacia el exterior. De tal manera que la energía calorífica de la biomasa que hace combustión dentro de la caldera pueda transformarse más eficientemente en otros tipos de energía según sean los requerimientos de la empresa, abaratando costos de producción y aumentando la productividad de la industria azucarera hondureña.

\section{MARCO TEÓRICO}

\section{A. Materiales refractarios}

Se conoce como material refractario a aquellos materiales cuyas propiedades permiten que pueda soportar temperaturas muy elevadas, sin embargo estos deben soportar una temperatura en específico sin sufrir ningún tipo de deterioro en sus condiciones internas, como por ejemplo la corrosión. Esta condición permite que los materiales refractarios sean utilizados en todos los hornos de fabricación, ya sea en las refinerías, industria química, metalurgia, industrias de vidrio y cerámica. El material refractario conocido por excelencia es la cerámica. Estos materiales están formados por partículas diminutas de óxido, las cuales están unidas a otros materiales de características refractarias pero más finas.

\section{B. Propiedades de los refractarios}

Las propiedades que estos materiales presentan, van a estar en función de su composición química. La relación alúmina sílice y los contenidos de impurezas, tales como los óxidos de hierro y los álcalis presentes $\left(\mathrm{Na}_{2} \mathrm{O}\right.$ y $\left.\mathrm{K}_{2} \mathrm{O}\right)$ van a determinar las temperaturas de fusión y la resistencia al ataque químico de los gases de combustión, especialmente en presencia de monóxido de carbono, libre y derivados de la combustión del azufre. 


\section{Densidad aparente de un material a granel}

La densidad $\varrho$ es la relación que hay entre la masa del refractario y el volumen. En la norma ASTM C134 [3] se llama bulk density. La densidad se expresa en libras por pie cúbico (pcf) o kilogramos por metro cúbico $\left(\mathrm{kg} / \mathrm{m}^{3}\right)$. La densidad en los refractarios es una medida indirecta de su capacidad calorífica o su habilidad de acumular calor.

\section{Porosidad aparente}

La porosidad aparente, también conocida como porosidad abierta, es la medida de los poros que están interconectados en la estructura del refractario. En la norma ASTM C830 [4] se llama Apparent Porosity. La porosidad tiene efecto directo en la habilidad del refractario de resistir la penetración de metales, escorias, gases y vapores. Por otra parte a mayor porosidad, mayor efecto aislante del refractario 1]. En muchas aplicaciones los refractarios son sometidos al impacto de piezas pesadas o al continuo paso de materiales sólidos como polvos y gases que los van erosionando. Para una mejor resistencia a estos efectos el material debe ser mecánicamente fuerte y tener una liga muy fuerte. Los ladrillos más fuertes generalmente muestran mejor resistencia a la abrasión. El módulo de ruptura y la resistencia a la compresión ofrecen el mejor indicador de la resistencia a la abrasión del refractario.

\section{E. Fuerza de trituración en frío y el módulo de ruptura de ladrillos}

La compresión es uno de los parámetros más usados para evaluar un refractario. La determinación en frío no puede ser usada para predecir su comportamiento en servicio, pero nos da una buena idea de cómo desarrolló su liga durante su fabricación. Los ladrillos más fuertes generalmente muestran mejor resistencia a la abrasión. El módulo de ruptura (Modulus of Rupture, MOR) y la resistencia a la compresión (Cold Crushing Strength, CCS) ofrecen el mejor indicador de la resistencia a la abrasión del refractario. La prueba de compresión en caliente es una medida de la capacidad del material de resistir los esfuerzos causados por la expansión térmica, los choques térmicos y en general los esfuerzos mecánicos a los que será sometido el refractario en operación. La compresión en caliente también nos sirve de indicador de la resistencia del material a la erosión y corrosión por metales y escorias junto con otras propiedades como la mineralogía y la porosidad.

El modulo de ruptura en frío (MOR) es otra medida de la resistencia del material, solo que esta es aplicada transversalmente para medir su resistencia a la flexión. Ambas pruebas tienen versiones en frío y en caliente. El módulo de ruptura y la resistencia a la compresión son descritas por la norma ASTM C133 [5]. La densidad, porosidad y resistencia a la compresión de los productos quemados dependen de muy variados factores, como la calidad de las materias primas, el tamaño de partícula y su empaquetamiento dentro de la estructura del material, la presión y la velocidad de prensado, la temperatura y el tiempo de quemado y la atmosferas del horno así como el tiempo y la velocidad de enfriamiento. Las cualidades de los refractarios no pueden ser determinadas solamente por unas cuantas de sus propiedades sino más bien de la correlación de todas sus propiedades.

A pesar del alto punto de fusión de la sílice de $1713^{\circ} \mathrm{C}$, su uso como refractario esta poco extendido debido a las dificultades que presenta en su manejo, porque la sílice es muy sensible a los cambios de temperatura.

\section{DETERMINACIÓN DE LA RESISTENCIA A LA ROTURA POR COMPRESIÓN Y POR FLEXIÓN EN FRÍO}

El procedimiento indicado en la norma ASTM C133 [5] consiste en someter los ladrillos y piezas refractarias a una carga de compresión y de flexión, en frío, para conocer la resistencia máxima admisible. Los aparatos o máquinas utilizados para realizar los ensayos de resistencia a la compresión y a la flexión deben tener características tales, que el error máximo de las presiones indicadas no sea superior al $2 \%$. Se usaron aparatos de la Universidad Nacional Autónoma de Honduras (UNAH) con el apoyo del laboratorio de ensayo de materiales de Instituto Nacional de Formación Profesional (INFOP).

\section{A. Fabricación del ladrillo refractario}

A continuación se presenta el procedimiento que se realizó para fabricar el ladrillo y asimismo someter las piezas a las diferentes pruebas para definir sus propiedades mecánicas. Se usaron tres tipos de cemento de la empresa Castwel Industries Nagpur (India): Supercast, Insulcast y Castocretesuper. En este estudio, se les asignan las letras $A, B$ y $C$ a estos tipos. De cada tipo, se fabricaron cinco ladrillos para cada uno de los dos ensayos. Los ladrillos se identifican con una letra del tipo y un número como subíndice, p.e. $A_{1}$. El cemento se fabricó en el mes de noviembre del 2013 y tiene una fecha de vencimiento nueve meses después. Lamentablemente, no fue posible conseguir cemento no vencido.

En primer lugar es necesario hacer referencia a las diferentes normativas que la ASTM presenta para la fabricación de los ladrillos refractarios esto implica realizar la respectiva traducción de la misma y si es necesario hacer referencia a el número de normativa para completar el procedimiento, esto permitirá crear o definir las características estándares que permitirán obtener resultados más confiables. A continuación se presentan el número de normativa a las cuales se hizo referencia en este documento:

1. C862-02 (Standard practice for Preparing Refractory Concrete Specimens by Casting) [2] 
2. C134-95 (Standard Test Methods for Size, Dimensional Measurements, and Bulk Density of Refractory Brick and Insulating Firebrick) [3]

3. C133-97 (Standard Test Methods for Cold Crushing Strength and Modulus of Rupture of Refractories)ASTM-C133

Ahora se procede a seleccionar el tamaño estándar de los ladrillos respectivamente de acuerdo a la prueba que será sometida. Para realizar esta investigación se tomaron las siguientes medidas:

- 2 pulgadas $\times 2$ pulgadas $\times 2$ pulgadas $(\mathrm{CCS})$

- 2 pulgadas $\times 2$ pulgadas $\times 9$ pulgadas $(\mathrm{MOR})$

Cuando se obtenga la mezcla es necesario previamente preparar los moldes según las normativas, se colocan los moldes en una superficie con un plástico y se le agrega aceite en las paredes del molde. Este le permitirá deslizar la pieza cuando se retire del molde. Se deposita la mezcla en el molde y se compacta la mezcla en el molde con una varilla. Esto elimina las burbujas de aire y crea una mezcla más compacta. Después de 5 horas de secado en el molde, se desmolda el ladrillo y se espera por 24 horas el secado total al ambiente. Se repiten los pasos anteriores para fabricar mas ladrillos y obtener una muestra más grande para analizar los resultados. Según la normativa, después los ladrillos se someten a otro proceso de secado en el horno a $110^{\circ} \mathrm{C}$ por 18 horas. Sin embargo, en este proceso se observó que en realidad es un proceso de evaporación del agua la cual está presente en el ladrillo y el objetivo es eliminarlo de la composición solida del ladrillo refractario ya fabricado. Al final, se toman las medidas de los ladrillos y se registra su masa. La precisión de la medida se da a partir de los moldes. Por eso es necesario que los moldes sean precisas para respetar las normativas que se están aplicando.

\section{B. El ensayo a la compresión}

Para determinar la resistencia a la rotura por compresión puede emplearse cualquier prensa hidráulica, con capacidad para alcanzar presiones superiores a las que se pueden prever en el ensayo.

En el ensayo a la compresión, la cara de apoyo y aquella en la cual se aplica la presión deben ser planas y paralelas entre sí. Si no lo son, se alisan aplicando una capa fina de gomalaca que se deja secar y luego una capa delgada de yeso de París, mediante una llana o lámina de vidrio, de tal modo que ambas caras sean planas y perpendiculares al eje longitudinal de la muestra de ensayo.

Cuando las caras menores del ladrillo o muestra de ensayo sean suficientemente planas y paralelas entre sí, se interponen entre las mismas y los apoyos de la prensa placas de material fibroceluloso, de aproximadamente $6 \mathrm{~mm}$ de espesor y que sobresalgan aproximadamente $50 \mathrm{~mm}$ de

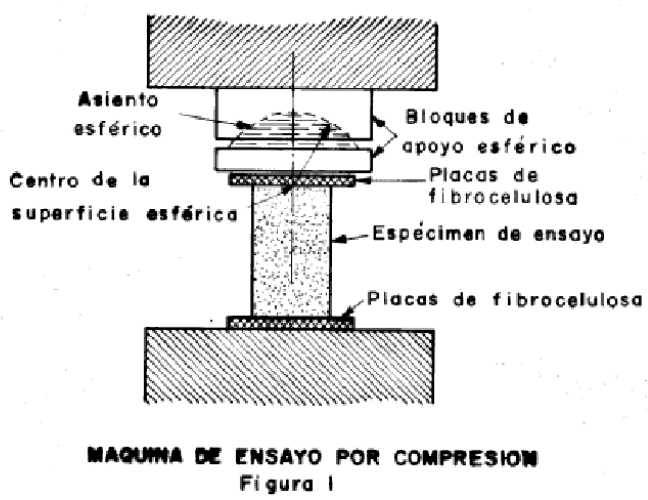

Figura 1: Máquina de ensayo por compresión [6, pág. 1]

los bordes de las caras de la muestra, como se muestra en la Fig. 1

Se toman el ancho y el espesor en milímetros de cada ladrillo o muestra, de acuerdo con lo indicado en la norma. En particular, se determina la densidad del ladrillo $\varrho$ mediante la fórmula:

$$
\varrho=\frac{d}{\ell w t}
$$

donde $\varrho$ es la densidad, $d$ es la masa seca del cemento (dry weight), $\ell, w$ y $t$ son la longitud (length), el ancho (width) y el grosor (thickness) del ladrillo.

Se coloca el ladrillo o muestra de ensayo con una de sus caras menores sobre el apoyo inferior de la prensa y hacer descender suavemente el superior, hasta obtener un perfecto asentamiento sobre la cara superior de la muestra asegurándose de que el eje de la barra transmisora de la presión coincida con el eje de la muestra de ensayo, como se muestra en la Fig. 1 .

Se aplica lentamente la presión. El aumento de la presión se prosigue hasta el momento de rotura de la muestra.

El cálculo de esfuerzo de compresión en frío $S$ está definido por la siguiente fórmula:

$$
S=\frac{F_{\text {máx }}}{A}
$$

donde $F_{\text {máx }}$ es la máxima carga total indicada en la máquina de prueba (en N) y $A$ es el área perpendicular a la linea de la carga del especimen $\left(\right.$ en $\mathrm{mm}^{2}$ ).

En tabla 1 se presentan los resultados obtenidos.

\section{El ensayo a la flexión}

- Tomar el ancho y el espesor, en centímetros, de cada ladrillo o muestra, de acuerdo con lo indicado en la norma.

- Colocar el ladrillo o muestra de ensayo con una de sus caras mayores sobre los apoyos de la prensa y 


\begin{tabular}{|c|c|c|c|c|c|c|c|c|}
\hline Ladrillo & $d$ & $\ell$ & $w$ & $t$ & $\varrho$ & $A$ & $F_{\text {máx }}$ & $S$ \\
\hline & $\mathrm{kg}$ & $\mathrm{mm}$ & $\mathrm{mm}$ & $\mathrm{mm}$ & $\left(\mathrm{g} / \mathrm{cm}^{3}\right)$ & $\mathrm{mm}^{2}$ & $\mathrm{~N}$ & $\mathrm{MPa}$ \\
\hline \hline$A_{1}$ & 0.265 & 50 & 50 & 53 & 2.00 & 2500 & 11400 & 4.56 \\
\hline$A_{2}$ & 0.27 & 53 & 53 & 50 & 1.92 & 2809 & 14300 & 5.09 \\
\hline$A_{3}$ & 0.26 & 53 & 53 & 50 & 1.85 & 2809 & 15700 & 5.59 \\
\hline$A_{4}$ & 0.265 & 53 & 53 & 50 & 1.89 & 2809 & 14000 & 4.98 \\
\hline$A_{5}$ & 0.265 & 53 & 53 & 50 & 1.89 & 2809 & 13900 & 4.95 \\
\hline \hline$B_{1}$ & 0.19 & 53 & 53 & 50 & 1.35 & 2809 & 12400 & 4.41 \\
\hline$B_{2}$ & 0.175 & 53 & 53 & 50 & 1.25 & 2809 & 8100 & 2.88 \\
\hline$B_{3}$ & 0.185 & 52 & 53 & 50 & 1.34 & 2756 & 11900 & 4.32 \\
\hline$B_{4}$ & 0.19 & 53 & 50 & 50 & 1.43 & 2650 & 19600 & 7.40 \\
\hline$B_{5}$ & 0.19 & 50 & 50 & 50 & 1.52 & 2500 & 5600 & 2.24 \\
\hline \hline$C_{1}$ & 0.27 & 53 & 53 & 50 & 1.92 & 2809 & 14100 & 5.02 \\
\hline$C_{2}$ & 0.27 & 53 & 53 & 50 & 1.92 & 2809 & 13700 & 4.88 \\
\hline$C_{3}$ & 0.28 & 53 & 53 & 50 & 1.99 & 2809 & 10100 & 3.60 \\
\hline$C_{4}$ & 0.28 & 53 & 50 & 50 & 2.11 & 2650 & 17800 & 6.72 \\
\hline$C_{5}$ & 0.275 & 52 & 52 & 53 & 1.92 & 2704 & 11100 & 4.11 \\
\hline
\end{tabular}

Tabla 1: Datos del ensayo de compresión en frío

\begin{tabular}{|c|c|c|c|c|c|c|c|c|}
\hline Ladrillo & $d$ & $\ell$ & $w$ & $t$ & $\varrho$ & $P$ & $L$ & $\mathrm{MOR}$ \\
\hline & $\mathrm{kg}$ & $\mathrm{mm}$ & $\mathrm{mm}$ & $\mathrm{mm}$ & $\mathrm{g} / \mathrm{cm}^{3}$ & $\mathrm{~N}$ & $\mathrm{~mm}$ & $\mathrm{MPa}$ \\
\hline \hline$A_{1}$ & 1.135 & 225 & 50 & 51 & 1.98 & 1100 & 139.7 & 0.0911 \\
\hline$A_{2}$ & 1.165 & 227 & 51 & 52 & 1.94 & 1600 & 139.7 & 0.1276 \\
\hline$A_{3}$ & 1.145 & 226 & 50 & 50 & 2.03 & 1300 & 139.7 & 0.1067 \\
\hline$A_{4}$ & 1.155 & 226 & 52 & 51 & 1.93 & 1300 & 139.7 & 0.1026 \\
\hline$A_{5}$ & 1.155 & 227 & 52 & 51 & 1.92 & 1100 & 139.7 & 0.0860 \\
\hline \hline$B_{1}$ & 0.895 & 225 & 55 & 53 & 1.36 & 1300 & 139.7 & 0.0978 \\
\hline$B_{2}$ & 0.855 & 225 & 50 & 55 & 1.38 & 900 & 139.7 & 0.0745 \\
\hline$B_{3}$ & 0.94 & 226 & 52 & 55 & 1.45 & 1200 & 139.7 & 0.0947 \\
\hline$B_{4}$ & 0.91 & 225 & 53 & 55 & 1.39 & 1200 & 139.7 & 0.0937 \\
\hline$B_{5}$ & 0.9 & 225 & 50 & 53 & 1.51 & 1000 & 139.7 & 0.0828 \\
\hline \hline$C_{1}$ & 1.21 & 227 & 51 & 51 & 2.05 & 1400 & 139.7 & 0.1116 \\
\hline$C_{2}$ & 1.21 & 227 & 50 & 48 & 2.22 & 1200 & 139.7 & 0.0976 \\
\hline$C_{3}$ & 1.255 & 223 & 52 & 51 & 2.12 & 1200 & 139.7 & 0.0972 \\
\hline$C_{4}$ & 1.245 & 229 & 50 & 52 & 2.09 & 1200 & 139.7 & 0.0959 \\
\hline$C_{5}$ & 1.245 & 229 & 50 & 53 & 2.05 & 1300 & 139.7 & 0.1039 \\
\hline
\end{tabular}

Tabla 2: Datos del ensayo de flexión en frío

hacer descender suavemente la pieza superior, transmisora de la presión, hasta obtener un asentamiento perfecto sobre la cara superior de la muestra. La pieza superior de la prensa debe abarcar el ancho de la muestra y aplicarse en el centro de la distancia entre apoyos, que debe ser el $80 \%$ de la longitud de la muestra, como se indica en la Fig. 2

- Aplicar lentamente la presión, hasta la rotura de la muestra.

El cálculo de esfuerzo de flexión en frío (MOR) está definido por la siguiente fórmula:

$$
\mathrm{MOR}=\frac{3 P L}{2 w \ell^{2}}
$$

dónde MOR es el esfuerzo de Flexión en frío (en MPa),
$P$ es la máxima carga total aplicada de rotura (en N), $L$ es la medida entre soportes (en mm), $w$ es el ancho del

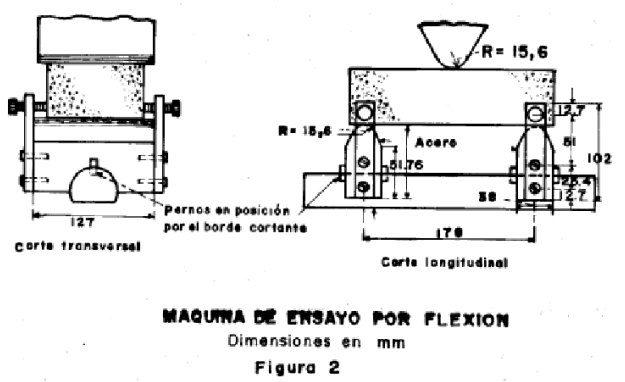

Figura 2: Máquina de ensayo por flexión [6, pág. 1] 


\begin{tabular}{|c||c|c||c|c|}
\hline Cemento & CSS/MPa & error $/ \mathrm{MPa}$ & $\mathrm{MOR} / \mathrm{MPa}$ & error $/ \mathrm{MPa}$ \\
\hline Supercast (A) & 5.034 & 0.37 & 0.1028 & 0.016 \\
\hline Insulcast (B) & 4.25 & 2.0 & 0.0887 & 0.0098 \\
\hline Concretesuper (C) & 4.866 & 1.2 & 0.1012 & 0.0066 \\
\hline
\end{tabular}

Tabla 3

especimen (en $\mathrm{mm}$ ) y $\ell$ su profundidad (en $\mathrm{mm}$ ).

En la tabla 2 se presentan los resultados obtenidos.

\section{Conclusiones}

- A simple vista se puede determinar que las propiedades como densidad eran diferentes cada uno de los cementos refractarios, y en base a otras propiedades se podría definir la calidad de cada uno de ellos.

- El proceso de fabricación de las probetas requiere de un elaborado método, ya que en base a estos parámetros los resultados pueden ser muy variables.

- En estas pruebas se tomó de referencia solamente 24 horas para secado natural de las piezas, suponemos que al aumentar este tiempo se brindara un cambio significativo en los resultados.

- Se observó que las propiedades de del cemento refractario en cuanto a textura, son variables en todos ellos. La textura de los cementos A y $\mathrm{C}$ era de un polvo más fina, y el $\mathrm{B}$ tenía un aspecto de granos o pequeñas piedras. Por lo tanto absorbía más agua en el momento de preparar la mezcla y pudo tener un efecto en la reducción de la resistencia.

- El conocer las diferentes normativas aplicables a estos tipos de pruebas mecánicas permiten ampliar el conocimiento y sobre todo el desarrollar investigación de campo de forma profesional, que brinden resultados más confiables y aceptables para dar soluciones a la industria que requiera el apoyo del estudio de los materiales.

- En base a los promedios de las resistencias obtenidas para cada cemento, se puede concluir que la mayor resistencia es del cemento A (Supercast); en segundo lugar ofreciendo una resistencia media está el cemento C (Castocrete Súper); y en tercer lugar ofreciendo una resistencia baja, ya sea por su composición química o por su fecha de vencimiento, es el cemento B (Insulcast).

\section{RECOMENDACIONES}

- Utilizar estos productos de acuerdo a la fechas estipuladas por el fabricante, respetando su fecha de caducidad.
- Para ampliar los criterios de selección del cemento se recomienda realizar un análisis térmico donde se determinen los coeficientes de transferencia de calor.

- Para evitar la variación de pico altos en las resistencias para cada una de los especímenes de un Cemento Refractario en específico, se aconseja utilizar un mayor número de especímenes para la muestra y de tal forma obtener un promedio más preciso.

\section{REFERENCIAS}

[1] American Society for Testing and Materials (ASTM). ASTM Cr04 / Cr04M - 15 Standard Test Method for Abrasion Resistance of Refractory Materials at Room Temperature. Disponible en http://www.astm.org/ Standards/C704.htm

[2] American Society for Testing and Materials (ASTM) (2008). ASTM C862 - 02(2008) Standard Practice for Preparing Refractory Concrete Specimens by Casting. Disponible en http://www.astm.org/ Standards/C862.htm

[3] American Society for Testing and Materials (ASTM) (2010). ASTM C134 - 95(2010) Standard Test Methods for Size, Dimensional Measurements, and Bulk Density of Refractory Brick and Insulating Firebrick. Disponible en http://www.astm.org/Standards/C134 htm

[4] American Society for Testing and Materials (ASTM) (2011). ASTM C830 - 00(2011) Standard Test Methods for Apparent Porosity, Liquid Absorption, Apparent Specific Gravity, and Bulk Density of Refractory Shapes by Vacuum Pressure. Disponible en http://www . astm.org/Standards/C830.htm.

[5] American Society for Testing and Materials (ASTM) (2015). ASTM C133 - 97(2015) Standard Test Methods for Cold Crushing Strength and Modulus of Rupture of Refractories. Disponible en http://www.astm.org/ Standards/C133.htm

[6] Instituto Ecuatoriano de Normalización (INEN) (1981). Materiales refractarios determinación de la resistencia a la rotura por compresión y por flexión en frío. Encontrado en https://law.resource.org/ pub/ec/ibr/ec.nte.0574.1981.pdf 